\title{
Future of Artificial Intelligence in Anesthetics and Pain Management
}

\author{
Harry McGrath1, Colin Flanagan², Liaoyuan Zeng³, Yiming Lei ${ }^{4}$ \\ ${ }^{1}$ Department of Anesthesiology, University Hospital Limerick, Limerick, Ireland \\ ${ }^{2}$ Department of ECE, University of Limerick, Limerick, Ireland \\ ${ }^{3}$ Department of Information and Communication Engineering, UESTC, Chengdu, China \\ ${ }^{4}$ Engineering Research Centre of Digital Hospital Systems, Peking University, Ministry of Education, Beijing, China \\ Email: mcgrath.har@gmail.com
}

How to cite this paper: McGrath, H., Flanagan, C., Zeng, L.Y. and Lei, Y.M. (2019) Future of Artificial Intelligence in Anesthetics and Pain Management. Journal of Biosciences and Medicines, 7, 111-118. https://doi.org/10.4236/jbm.2019.711010

Received: May 24, 2019

Accepted: November 10, 2019

Published: November 13, 2019

\begin{abstract}
The potential of the second wave of Artificial Intelligence (AI) to change our lives beyond recognition is both exciting and challenging. AI has been around for over three decades, and this new approach of artificial intelligence, due to enhancements in technology, both software, and hardware, has resulted in the fact that human decision-making is considered inferior and erratic in many fields: none more so than medicine. Machine learning algorithms with access to large data sets can be trained to outperform clinicians in many respects. AI's effectiveness in accurate diagnosis of various medical conditions and medical image interpretation is well documented. Modern AI technology has the potential to transform medicine to a level never seen before in terms of efficiency and accuracy; but is also potentially highly disruptive, creating insecurity and allowing the transfer of expert domain knowledge to machines. Anesthetics is a complex medical discipline and assuming AI can easily replace experienced and knowledgeable medical practitioners is a very unrealistic expectation. AI can be used in anesthetics to develop, in some respects, more advanced clinical decision support tools based on machine learning. This paper focuses on the complexity of both AI developments, deep learning, neural networks, etc. and opportunities of $\mathrm{AI}$ in anesthetics for the future. It will review current advances in AI tools and hardware technologies as well as outlining how these can be used in the field of anesthetics.
\end{abstract}

\section{Keywords}

Anesthesiology, Machine Learning, Pain Management

\section{Introduction}

Anesthesia can be sub-divided into four general areas: anesthesia, intensive care 
medicine, critical emergency medicine, and pain medicine. The development of this field of medicine has a long history, but essentially the concept has not changed. The purpose is to reduce sensitivity allowing surgery to be performed.

Early anesthetics used narcotics (opium) alcohol and belladonna. Dr. William Morton (1846) removed a tumor from the jaw of a patient using ether to render the patient unconscious. Many of these early anesthetics, such as nitrous-oxide and chloroform had significant side effects. Anesthesia is a very specialized aspect of medicine, where clinicians are trained in all aspects of physiology, Physics, and pharmacology, as well as medicine in general with a particular focus on those aspects which may impact on a surgical procedure [1].

Pre-operative and post-operative are critical aspects of patient monitoring. Drug control management during surgery requires very accurate and controlled does to be administered in a timely fashion. Any errors in any aspect of the process are extremely dangerous. Intensive care requires support for numerous possible medical intervention cardiovascular instability, airway or respiratory compromise acute renal failure, or multiple organ dysfunction syndrome. After major surgery monitored patient vitals is critical in the intensive care unit. Medications used to treat chronic painful conditions need to be carefully monitored and most medications have significant side effect profiles. Thus much of pain medicine treatment is theatre-based interventional techniques which require a specific skill set [2].

\section{Data Analytics}

Modern medical practice, and particularly that within the hospital environment, has been under intense scrutiny in an attempt to improve patient safety and optimize outcomes [3]. The ASA score physical status classification system is a system for assessing the fitness of patients before surgery. In 1963 the American Society of Anesthesiologists adopted the five-category physical status classification system; a sixth category was later added [4] [5] (Table 1).

\section{AI in Medicine}

With the growing excitement of AI technology applications are being developed in many areas previously thought to be the sole domain of human intelligence. This trend is especially obvious in the fields of healthcare and medicine, which have always been an emotional and sensitive area for people. Enthusiastic proponents of AI claim that machines can outperform humans in many areas of medical diagnosis. However, one may ask, how can patients be convinced that a machine diagnosis is more reliable than that of a human physician?

One of the major concerns is the Trust and confidence in the use of sensitive data: In many sectors (in particular those serving individual people directly), data needs to be protected, for reasons of privacy, security, confidentiality, and commercial sensitivity. Existing programmes have been complex to arrange, and some have attracted criticism, such as DeepMind's collaboration with the Royal 
Table 1. ASA classification score.

\begin{tabular}{cl}
\hline ASA 1 & $\begin{array}{l}\text { Anormal healthy patient (BM under 30), a non-smoking patient with good } \\
\text { exercise tolerance. }\end{array}$ \\
ASA 2 & $\begin{array}{l}\text { A patient with mild systemic disease. Patient with no functional limitations } \\
\text { and a well-controlled disease. }\end{array}$ \\
ASA 3 & $\begin{array}{l}\text { A patient with a severe systemic disease that is not life-threatening Patient } \\
\text { with aome functional limitation as a result of disease }\end{array}$ \\
ASA 4 & $\begin{array}{l}\text { A patient with a severe systemic disease that is a constant threat to life. Patient } \\
\text { with fictional limitation from life-threatening disease. }\end{array}$ \\
ASA 5 & $\begin{array}{l}\text { A moribund patient who is not expected to survive. The patient is not expected } \\
\text { to survive beyond the next 24 hours without surgery. } \\
\text { Abrain-dead patient whose organs are being removed with the intention of } \\
\text { transplanting them into another patient. }\end{array}$ \\
\hline
\end{tabular}

Free Hospital. In Europe, the stringent provisions of the recently enacted General Data Protection Regulation (GDPR) emphasise this point [6].

\subsection{Artificial Intelligence}

A principal subcategory of $\mathrm{AI}$ is Machine Learning (ML), where algorithms learn from data to make decisions or predictions. So-called Deep Learning is a subcategory of ML, In Deep Learning, a computational structure has used that attempts to mimic, in a crude way, the architecture of the human brain. This structure is the Artificial Neural Network (ANN), comprised of "layers" of simple elements (mimicking neurons in the cerebral cortex) connected in a network structure. In popular media, deep learning and AI are presented as something new, in reality, many of the ideas have been commonplace for 40 or more years. The recent dramatic breakthroughs inability of $\mathrm{AI}$ is almost wholly attributable to advances in computer hardware that have made much larger ANNs (i.e., "deep nets") feasible [7] (Figure 1).

Technology, software, and tools for AI are a big business and it not surprising that the large Technology companies such as Google (Google Cloud AutoML), Amazon, Microsoft (Azure ML studio) are offerings in Machine Learning solutions. Even though AI is considered very complex, these companies provide visual programming for machine learning models to create simplified solutions to various applications. One of the leading AI libraries for machine learning is Tensor Flow developed by Google for applications of deep learning neural networks [8].

An immediate question is: what distinguishes different neural networks? Principally, it is the number of layers of neurons in the network. Here, for example, we see a network with five layers, one input, three internal ("hidden") and one output. Inputs (sensor data, images) are presented at the inputs, and the desired outputs appear at the output layer.

It is commonly recognized that the more hidden layers there are in a neural network, the more powerful it is. The original ("shallow") neural networks of the 80 's and 90's had one or two hidden layers, restricted by computer hardware 


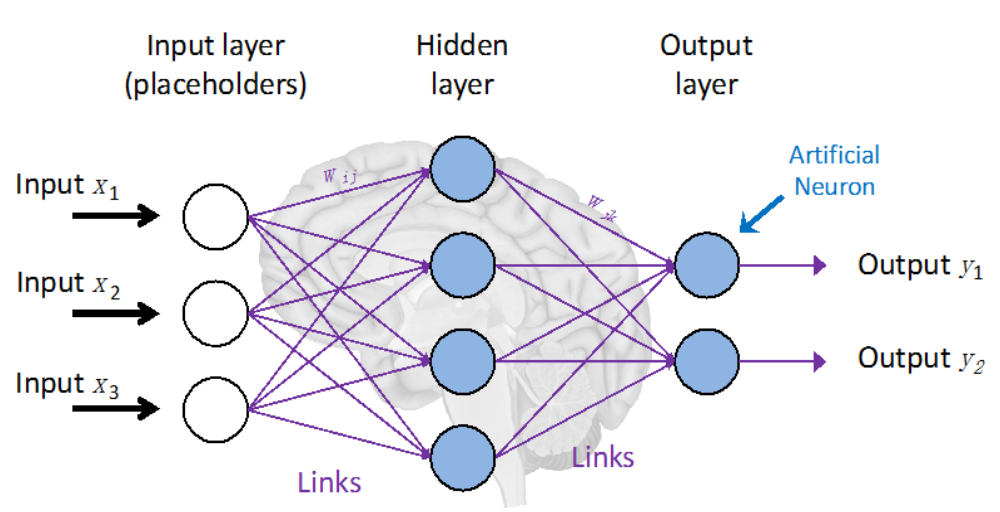

Figure 1. Basic neural network design.

limitations. The deep networks of the current century have rapidly advanced to have dozens of layers (hundreds are not uncommon). Generally, a network is regarded as deep if it has six or more layers (Figure 2).

What makes ANNs different from typical computer programs is that they are trained, rather than programmed. A neural network architect designs the network, specifies it to a system like TensorFlow, then trains it with a large set of data that specifies input and desired responses. The network learns by applying an error minimization procedure (“Gradient Descent") that tends, over time, to produce the desired mapping between inputs and outputs that is implicit in the training set. The more extensive the network, the larger the training set needed; modern training sets can be 100's of gigabytes in size. Moreover, the larger the network and training set, the longer the network takes to train. Reported training times of weeks on large computational clusters are not uncommon [9].

The difficulty of AI and machine learning models is the classifier and training dataset used. The performance is essential to minimize the prediction error, this directly relates to having a large dataset. Difference between the actual output and predicted output is the error. Based on the performance of different models we choose the model which ranks highest in performance. The Error is a summation of reducible and irreducible error. The reducible Error is bias and Variance. Bias is how far are the predicted values from the actual values. If the average predicted values are far off from the actual values then the bias is high. Variance occurs when the model performs well on the trained dataset but does not do well on a dataset that it is not trained on, like a test dataset or validation dataset. Variance occurs when the model performs well on the trained dataset but does not do well on a dataset that it is not trained on, Variance tells us how scattered is the predicted value from the actual value [10] (Figure 3).

Data sets that could be used to develop specific new AI applications in high-value sectors maybe be used, due to security and confidentiality and AI cannot be applied to challenges in these sectors. This is particularly apparent in healthcare, which is also an area where AI can add great value. The obstacle to AI implementation in healthcare is not technological but access to data. Research is hampered by difficulties in accessing large medical datasets, for legal or other reasons. 


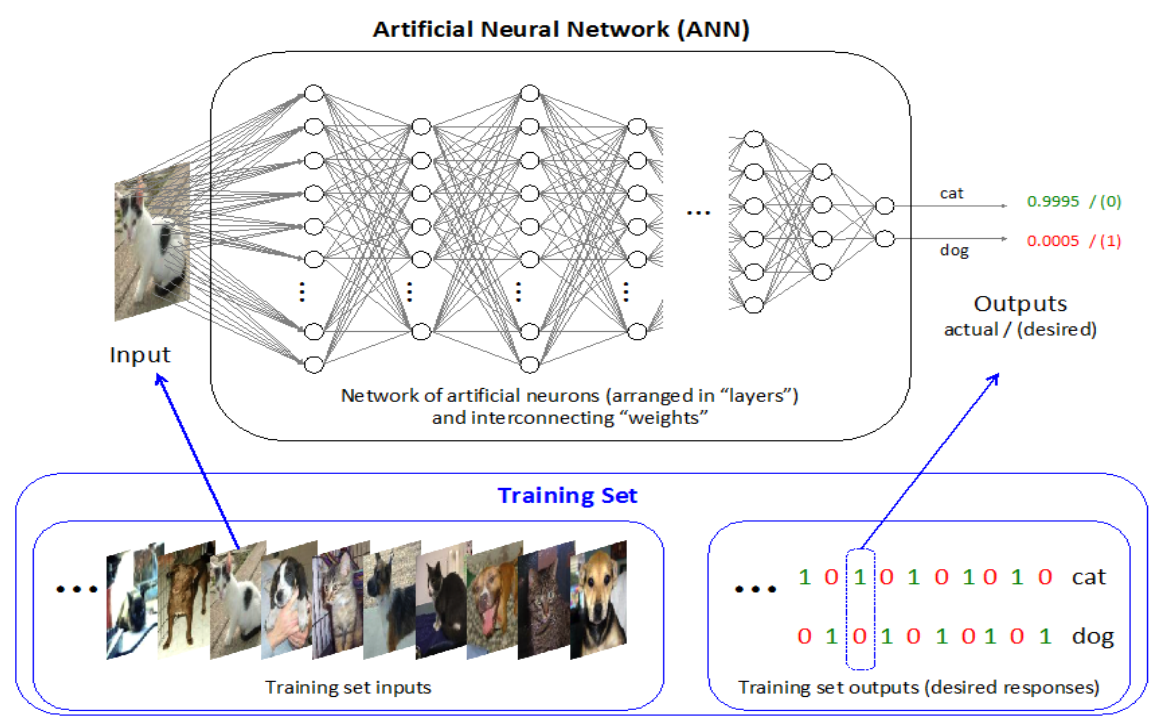

Figure 2. Multi-layered, backpropagation neural network.

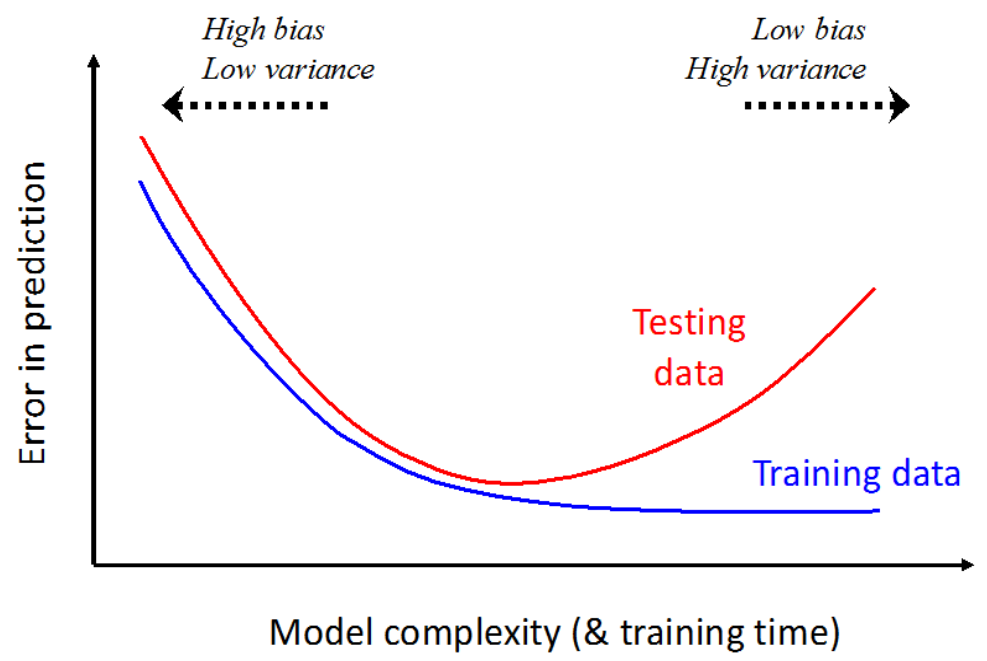

Figure 3. Bias vs variance.

However, for ML models it is the labeled data that is the most precious commodity. Modern ML models require large amounts of task-specific training data, and creating these labels by hand is often too slow and expensive. The most significant thrust to intelligent computing has come from the availability of real data. A key element we are missing is predictive (or unsupervised) learning: "the ability of a machine to model the environment, predict possible futures and understand how the world works by observing it and acting in it". Hence, it is imperative to understand the data, variations, and behaviour [11].

In Supervised Learning input data or training examples come with a label, and the goal of learning is to be able to predict the label for new, unforeseen examples. Labeling the data is expensive and error-prone. Data quality issues can lead to "garbage in, garbage out" in machine learning. We need several experts to label the same image to ensure the correctness of the diagnosis, and hence acquir- 
ing a dataset for the given medical task would be several times the amount it takes to annotate a single image. The problem is even more difficult in traditional enterprise settings because of data sparsity, data quality and lack of domain experts.

\subsection{Anaesthetics Benefits of AI}

Anesthesiologists focus not just on administering anesthetics during the surgical procedure itself, but identify high-risk patients and optimize their fitness for surgery as well as afterwards in order to promote and enhance recovery. Other functions include major trauma, resuscitation, airway management, and critical emergencies that pose an immediate threat to life. The main Areas that AI will influence Anesthestics will be:

1) Classification of ASA Score

2) Monitoring and Control of Drug Dosage

3) General Data Collection of Post and Pre-Operative Data

\subsubsection{Classification of ASA Score}

American Society of Anaesthesiologists' (ASA) classification is the assessment system used for preoperative surgical patients, variations of this grading system are a common clinical problem. The difficulty of ASA score is due to anesthesiologists interpretation, based on other factors to indicate operative risk such as the type of surgery, age, anaemia, obesity, and with patients who have recovered from a myocardial infarction. Geriatric patients, even in the absence of disease, are far more "fragile" in their tolerance of anesthetics compared to young patients. The specific correlation of ASA scores with operating times, hospital length of stay, postoperative infection rates, overall morbidity and mortality rates following gastrointestinal, cardiac, and genitourinary surgery has also been extensively studied [12] [13] [14]. The human error factor and Variance of the ASA score allows for AI and Deep learning Algorithms to support and guide clinicians in more accurate and consistent allocation to this grading system.

\subsubsection{Monitoring and Control of Drug Dosage}

Anaesthesia is a balance between the amount of anaesthetic drug(s) administered and the state of arousal of the patient. Anesthesiology function is to provide safe, effective, and efficient environment to patients under surgery. This process involves the delivery and monitoring of targeted control drugs. Target-controlled infusion (TCI) is part of anesthesia delivery in many countries, resulting in the precision, reliability, efficacy, and safety of IV anesthesia delivery. Drug innovations are primarily focused on modifying the chemical structures of existing drugs to improve their pharmacodynamic, pharmacokinetic, and side effect properties [15] [16].

Depth of anaesthesia monitors is subjective and depends on patients classification and surgery type to allow accurate drug administration against the measured state of arousal of the patient. Anaesthesia monitoring using EEG or 
mid-latency auditory-evoked potentials is commercially available as surrogate measures of anaesthetic drug effect. The variation of patients sensitivity The intensity of surgical stimulation varies throughout surgery, and the haemodynamic effects of the anaesthetic drugs may limit the amount that can be given safely, it is not uncommon for there to be critical imbalances between anaesthetic requirement and anaesthetic drug administration. Under dosing may be because of equipment failure or error may occur. Conversely, inappropriate titration of the hypnotic components, leading to an excessive depth of anaesthesia (DoA), might compromise patient outcome [17] [18].

\subsubsection{General Data Collection of Post and Pre-Operative Data}

The world of AI is based on having large data sets that will allow machines to perform repetitive and accurate analysis. In anaesthetics the collection of pre-and post-operative information concerning patients and surgical procedures will provide an excellent controlled environment where efficiencies errors can be reduced can be achieved. Storage, security and use of this information are something that is of concern in only health care system.

\section{Conclusion}

A major issue with current deep learning systems is "opacity." Although a machine may be trained to perform a specific input-output mapping, it is often unclear as to which part of the training network is responsible for any specific outcome. This is undesirable, as physicians need to understand and trust the operation of any autonomous anaesthesia system. In particular, a physician needs to be sure that the machine will not generate "wild" responses in unforeseen operating conditions. Currently, acceptable methods solve this by increasing the training sets and more complex levels of processing, without a precise analysis of cognitive bias that may occur. Decision making for use in anaesthetics cannot be a nondeterministic closed process, and clinicians need to have full control and understanding of the decisions developed by these rule-based algorithms. Anaesthetics involves much cognitive and dexterity based work. Technology is increasingly encroaching on both of these areas. AI Systems using Machine based Learning tools and software can be very useful in some aspects of clinical decisions within anaesthetics.

\section{Conflicts of Interest}

The authors declare no conflicts of interest regarding the publication of this paper.

\section{References}

[1] Neill, D.B. (2013) Using Artificial Intelligence to Improve Hospital Inpatient Care. IEEE Intell Syst, 28, 92-95. https://doi.org/10.1109/MIS.2013.51

[2] (2018) Professionalism in Anaesthesia, Intensive Care and Pain Medicine. College of Anaesthetists of Ireland. 
[3] Lanier, W.L. (2006) A Three-Decade Perspective on Anesthesia Safety. The American Surgeon, 72, 985-989.

[4] Knuf, K.M., Maani, C.V. and Cummings, A.K. (2018) Clinical Agreement in the American Society of Anesthesiologists Physical Status Classification. Perioper Med (Lond), 7, 14. https://doi.org/10.1186/s13741-018-0094-7

[5] Kraev, A.I., et al. (2018) Improving the Power of the American Society of Anesthesiology Classification System to Risk Stratify Vascular Surgery Patients Based on National Surgical Quality Improvement Project-Defined Functional Status. Annals of Vascular Surgery, 52, 153-157. https://doi.org/10.1016/j.avsg.2018.04.005

[6] Hall, W. and Pesenti, J. (2017) Growing the Artificial Intelligence Industry in the UK. Department for Digital, Culture, Media \& Sport and Department for Business, Energy \& Industrial Strategy. Part of the Industrial Strategy UK and the Commonwealth.

[7] Jain, A.K., Mao, J.C. and Mohiuddin, K.M. (1996) Artificial Neural Networks: A Tutorial. Computer, 3, 31-44. https://doi.org/10.1109/2.485891

[8] Patel, V.L., Shortliffe, E.H., Stefanelli, M., et al. (2009) The Coming of Age of Artificial Intelligence in Medicine. ArtifIntell Med, 46, 5-17.

https://doi.org/10.1016/j.artmed.2008.07.017

[9] Hastie, T., Tibshirani, R. and Friedman, J. (2009) The Elements of Statistical Learning. Data Mining, Inference, and Prediction. 2nd Edition. Springer.

[10] Khandelwal, R. (2018) Bias and Variance in Machine Learning.

[11] Murdoch, T.B. and Detsky, A.S. (2013) The Inevitable Application of Big Data to Health Care. JAMA, 309, 1351-1352. https://doi.org/10.1001/jama.2013.393

[12] Dilsizian, S.E. and Siegel, E.L. (2014) Artificial Intelligence in Medicine and Cardiac Imaging: Harnessing Big Data and Advanced Computing to Provide Personalized Medical Diagnosis and Treatment. Curr Cardiol Rep, 16, 441. https://doi.org/10.1007/s11886-013-0441-8

[13] Howard, R., Yin, Y.S., McCandless, L., Wang, S., Englesbe, M. and Machado-Aranda, D. (2019) Taking Control of Your Surgery: Impact of a Prehabilitation Program on Major Abdominal Surgery. J. Am. Coll. Surg, 228, 72-80. https://doi.org/10.1016/j.jamcollsurg.2018.09.018

[14] Ridgeway, S., Wilson, J., Charlet, A., Pearson, A. and Coello, R. (2005) Infection of the Surgical Site after Arthroplasty of the Hip. J Bone Joint Surg Br, 87, 844-850. https://doi.org/10.1302/0301-620X.87B6.15121

[15] Mahmoud, M. and Mason, K.P. (2018) Recent Advances in Intravenous Anesthesia and Anesthetics. F1000Research, 7. https://doi.org/10.12688/f1000research.13357.1

[16] Mahmoub, M. (2018) Recent Advances in Intravenous Anesthesia and Anesthetics. PMC5911929, PMCID.

[17] Bruhn, J., Myles, P.S., Sneyd, R. and Struys, M.M.R.F. (2006) Depth of Anaesthesia Monitoring: What's Available, What's Validated and What's Next? BJA: British Journal of Anaesthesia, 97, 85-94. https://doi.org/10.1093/bja/ael120

[18] Schnider, T.W., Minto, C.F., Gambus, P.L., Andresen, C., Goodale, D.B., Shafer, S.L. and Youngs, E.J. (1998) The Influence of Method of Administration and Covariates on the Pharmacokinetics of Propofol in Adult Volunteers. Anesthesiology, 88, 1170-1182. https://doi.org/10.1097/00000542-199805000-00006 